\title{
The influence of irrigation fluid temperature on recurrence in the evacuation of chronic subdural hematoma
}

\author{
Andreas Bartley ${ }^{1,2}$ (D) Asgeir S. Jakola ${ }^{1,2} \cdot$ Magnus Tisell $^{1,2}$
}

Received: 29 September 2019 / Accepted: 18 November 2019 / Published online: 4 December 2019

(C) The Author(s) 2019

\begin{abstract}
Background Chronic subdural hematomas (cSDH) are one of the most common conditions requiring neurosurgical treatment. The reported recurrence after surgery is $3-21.5 \%$ with closed system drainage. In clinical practice, irrigation fluids at body temperature $\left(37^{\circ} \mathrm{C}\right)$ and at room temperature $\left(22^{\circ} \mathrm{C}\right)$ are routinely used in the evacuation of cSDH. Our hypothesis was that irrigation at body temperature might have more beneficial effects on coagulation and solubility of the chronic subdural hematoma than irrigation at room temperature. The aim of this study was to compare the effects of different intraoperative irrigation fluid temperatures on recurrence rates.

Methods This was a retrospective study where we included all consecutive patients from a defined geographical area of western Sweden between September 2013 and November 2014. In the course of 6 months, we performed intraoperative irrigation at body temperature $\left(37^{\circ} \mathrm{C}\right.$, BT-group) during burr hole evacuation of chronic subdural hematoma. This was then compared with the previous 6-month period, when irrigation fluid at room temperature $\left(22^{\circ} \mathrm{C}\right.$, RT-group) was used. The primary endpoint was same-sided recurrence in need of reoperation within 6 months.

Results Recurrence occurred in 11 of 84 (13.1\%) patients in the RT-group compared with 4 of 88 (4.5\%) in the BT-group ( $p=$ 0.013). There were no significant between-group differences regarding age, sex, duration of surgery, frequency of bilateral hematomas, hematoma density, and use of anticoagulant/antithrombotic therapy.

Conclusion Our study demonstrates that intraoperative irrigation fluid at body temperature is associated with lower recurrence rates compared with irrigation fluid at room temperature. To investigate this further, a prospective randomized controlled trial has been initiated (clinicaltrials.gov, NCT0275235).

Trial registration ClinicalTrials.gov Identifier: NCT0275235
\end{abstract}

Keywords Chronic subdural hematoma $\cdot$ Irrigation fluid $\cdot$ Temperature $\cdot$ Surgical evacuation $\cdot$ Recurrence

\section{Abbreviations \\ cSDH Chronic subdural hematoma \\ BT Body temperature \\ RT Room temperature}

This article is part of the Topical Collection on Brain trauma

Andreas Bartley

andreas.bartley@vgregion.se

1 Department of Neurosurgery, Sahlgrenska University Hospital, Blå stråket 5, 41345 Gothenburg, Sweden

2 Institute of Neuroscience and Physiology, Department of Clinical Neuroscience, University of Gothenburg, Sahlgrenska Academy, Box 430, 40530 Gothenburg, Sweden

\section{Introduction}

Surgical evacuation of chronic subdural hematoma (cSDH) is one of the most common neurosurgical procedures. The incidence of $\mathrm{cSDH}$ is expected to increase due to the widespread use of anticoagulant/antithrombotic therapy together with an anticipated longer life expectancy in the general population [1, $2,14]$. Data from the Swedish National Board of Health show that the number of surgeries for $\mathrm{cSDH}$ has doubled in Sweden in the last 18 years [13].

The estimated recurrence of cSDH after surgical evacuation of $\mathrm{cSDH}$ is $3-21.5 \%$ with closed system drainage [16]. Reduction of recurrence rates may not only lessen the future load on the health care system but may also decrease the morbidity and mortality associated with recurrence $[6,10]$. 
The most commonly used surgical procedure for $\mathrm{cSDH}$ treatment is evacuation via one or more cranial burr holes combined with postoperative drainage [8]. The procedure is often combined with perioperative irrigation of the subdural space. Although the benefits of perioperative irrigation have not been shown in a controlled study, there is evidence in favor of irrigation $[7,9]$.

A poll conducted on the neurosurgical networking site www.neurosurgic.com (accessed on 25th of September 2017) showed that out of 620 respondents, $57 \%$ used irrigation fluid at body temperature $\left(37^{\circ} \mathrm{C}\right), 40 \%$ used irrigation fluid at room temperature $\left(22{ }^{\circ} \mathrm{C}\right)$, and $3 \%$ did not use irrigation at all. Thus, there was a large variation in practice concerning the temperature of the irrigation fluid. In theory, the temperature of the irrigation fluid might have an impact on recurrence, possibly due to improved coagulation and solubility of the $\mathrm{cSDH}$ when performing irrigation at body temperature versus irrigation at room temperature $[4,15]$. However, no studies have evaluated the potential clinical impact of irrigation fluid temperature in the evacuation of $\mathrm{cSDH}$.

The aim of this study was to explore whether or not the temperature of the irrigation fluid has an impact on recurrence rates. More specifically, we compared irrigation fluid at room temperature (RT-group) with irrigation fluid at body temperature (BT-group).

\section{Materials and methods}

\section{Study design}

Sahlgrenska University Hospital is the sole provider of neurosurgical care in western Sweden. The hospital has a welldefined geographical catchment area of about 1.8 million inhabitants. Approximately, 150-190 surgeries for cSDH are performed at our department annually. In the course of 6 months, between September 2013 and November 2014, we performed intraoperative irrigation at body temperature $\left(37^{\circ} \mathrm{C}\right)$ during burr hole evacuation of $\mathrm{cSDH}$. Consecutive patients from this period were then compared with consecutive patients operated during the previous 6-month period when room temperature $\left(22^{\circ} \mathrm{C}\right)$ irrigation fluid was used. All patients had a postoperative follow-up of 6 months. The primary endpoint was recurrence requiring reoperation within 6 months. Secondary endpoints were complications requiring hospital admission and mortality.

\section{Surgical technique}

The hematoma was evacuated via one or two burr holes combined with perioperative irrigation with Ringer's lactate followed by active subgaleal drainage [5, 12]. The surgery was performed either under general or local anesthesia. The patient was kept in the supine position until the drain was removed the day after surgery. This surgical technique was adopted by all surgeons at our department.

\section{Postoperative management}

Typically, most patients were sent to their local hospital the day after surgery. We do not routinely perform a postoperative CT scan at our department prior to discharge, except in the absence of improvement after surgery [11]. This policy was similar in both time periods. In total, $63 \%$ of the BT-group and $60 \%$ of the RT-group had at least one postoperative $\mathrm{CT}$ scan performed during the follow-up period. If the patient developed recurrent neurological symptoms (headache, hemiparesis, dysphasia, etc.), a CT scan at the local hospital was recommended and our department was contacted subsequently. We did not focus on minor morbidity during the postoperative follow-up but instead on mortality and morbidity requiring hospital admission. There was no change in postoperative management during the study period.

\section{Statistical analysis}

The primary endpoint was analyzed with the $\chi^{2}$ test for frequency comparison. Secondary endpoints were analyzed with the $\chi^{2}$ test for categorical data. Normally distributed numerical data was analyzed with Student's $t$ test and the Mann-Whitney $U$ test if skewed. Statistical significance was set at $5 \%$.

\section{Ethical approval}

The Regional Ethics Committee in Gothenburg, Sweden, reviewed this study and concluded that no formal approval was necessary due to its retrospective observational character.

\section{Results}

Table 1 shows the baseline characteristics for the two groups. No statistically significant differences were seen between the groups regarding age, sex, anticoagulant/antithrombotic therapy, bilateral hematomas, hematoma density, maximum hematoma width, or duration of surgery.

Recurrence within 6 months occurred in 11 of $84(13.1 \%)$ in the RT-group compared with 4 of $88(4.5 \%)$ in the BTgroup ( $p=0.013$ ) (Fig. 1). All but one recurrence occurred within 2 months of surgery.

In total, three cases of mortality in the RT-group and four cases in the BT-group were noted during the postoperative follow-up period of 6 months. In the RT-group, one patient had a postoperative intracerebral hematoma 
Table 1 Characteristics of the compared groups. $B T$ body temperature, $R T$ room temperature

\begin{tabular}{llll}
\hline Variables & BT-group; $n=88$ & RT-group; $n=84$ & Difference \\
\hline Mean age (SD) & $74.5(12)$ & $75.1(13)$ & $p=0.77$ \\
No. of females (\%) & $21(24)$ & $24(28)$ & $p=0.27$ \\
Mean duration of surgery (SD) & $44 \mathrm{~min}(20.6)$ & $46 \mathrm{~min}(15.1)$ & $p=0.84$ \\
No. of patients treated with anticoag/antitromb (\%) & $40(45)$ & $40(47)$ & $p=0.54$ \\
Mean maximal hematoma width (SD) & $19 \mathrm{~mm}(7.1)$ & $18 \mathrm{~mm}(6.7)$ & $p=0.34$ \\
CT image: hypodense (\%) & $38(43)$ & $35(42)$ & $p=0.60$ \\
CT image: isodense (\%) & $25(28)$ & $19(24)$ & $p=0.16$ \\
CT image: hyperdense (\%) & $10(12)$ & $11(13)$ & $p=0.51$ \\
CT image: membranous (\%) & $15(17)$ & $18(21)$ & $p=0.26$ \\
Bilateral hematoma (\%) & $18(20)$ & $15(18)$ & $p=0.43$ \\
\hline
\end{tabular}

and died within 30 days. In the BT-group, one patient suffered a basal ganglia infarction within 30 days, and another died of unknown cause at the local hospital 10 days after surgery. The remaining cases of mortality in both groups all occurred more than 2 months after surgery of causes unrelated to the surgery. If considering the postoperative hematoma and death of unknown cause as recurrences, the recurrence rates would be $14.2 \%$ for the RT-group and $5.6 \%$ for the BT-group, respectively $(p=0.016)$.

In addition to mortality, we also recorded complications leading to hospital admission during the follow-up period. In the BT-group, we recorded 1 case each of pneumonia, urinary tract infection, and non-ST elevation myocardial infarction (NSTEMI). In the RT-group we recorded 1 case each of wound infection and postoperative seizure. In addition, there were two cases (one in each group) of acute subdural hematomas requiring surgery. Furthermore, there were two cases of symptomatic pneumocephalus (one in each group), but neither required surgical intervention.

\section{Discussion}

Our results show a statistically significant reduction in recurrence rates, from 13.1 to $4.5 \%$, when using irrigation fluid at body temperature versus irrigation fluid at room temperature. There were no statistically significant differences in mortality or overall complication frequency between the two groups. To the best of our knowledge, this is the first study comparing the effects of different irrigation fluid temperatures used in the evacuation of $\mathrm{cSDH}$.

Our finding indicates that the irrigation fluid temperature may affect recurrence rates. Theoretically, this might be explained by a negative effect on coagulation when using irrigation fluid at room temperature [15]. It is also possible that
Fig. 1 Frequency of recurrence between groups (BT body temperature, $R T$ room temperature)

\section{Recurrence rate of chronic subdural hematoma (\%)}

$$
p=0.013
$$

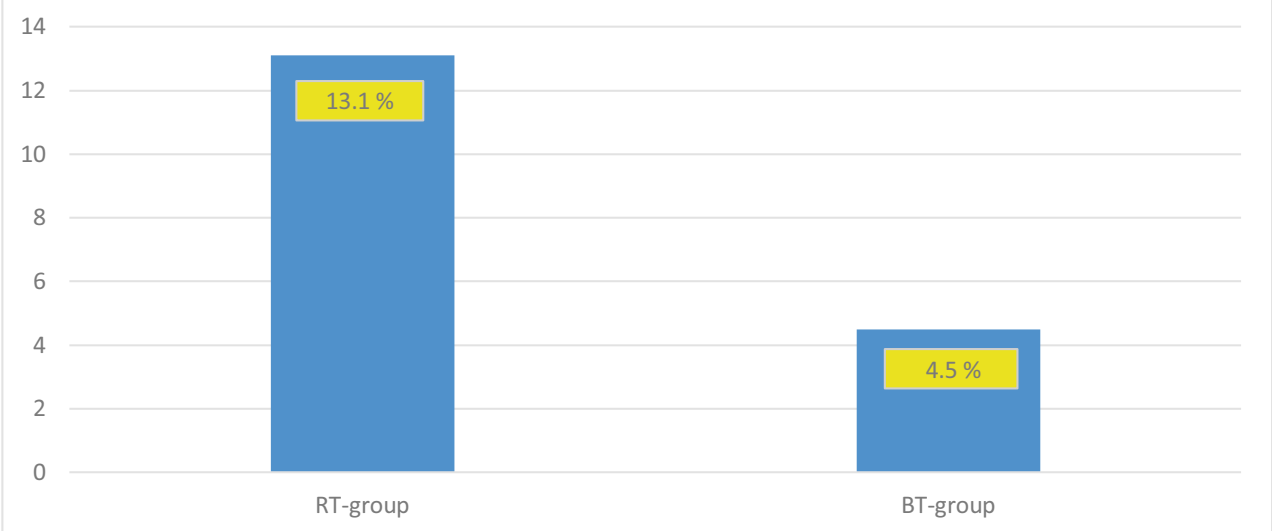


irrigation fluid at body temperature might increase the solubility of the cSDH, thereby facilitating evacuation [4].

Due to the expected increase in frequency of surgeries for $\mathrm{cSDH}$, it is of great importance to try to optimize treatment strategies with evidence-based treatments $[1,2,13,14]$.

The strengths of this study are that the patients were included consecutively from a defined geographical area. Furthermore, digitalized medical records used at our department, as well as at the local hospitals in our population-based catchment area, enabled complete follow-up regarding mortality, recurrence, and hospital admissions. All cases of recurrence requiring surgery were treated at our department. Finally, one surgical technique was used when evacuating cSDH.

Limitations of the study include the retrospective design. Furthermore, the possibility of individual surgeons changing their surgical behavior when a study or a new intervention is undertaken cannot be overlooked, such as during the 6-month period when we changed our surgical routine to using irrigation fluid at body temperature. To address this matter, we used the duration of surgery as a surrogate marker for increased length of irrigation, which did not differ between groups.

\section{Conclusions}

Our study shows that perioperative irrigation fluid at body temperature is associated with significantly lower recurrence rates compared with irrigation fluid at room temperature. In order to further investigate the results of this study, we have initiated a prospective randomized controlled trial (clinicaltrials.gov, NCT0275235) [3].

Funding information Open access funding provided by University of Gothenburg. This study is funded by a regional R\&D grant (Västra Götalandsregion, Sweden) VGFOUREG-655761, the Hjalmar Svensson research grant (HJSV2016023), and the Edith Jacobsson research grant (no applicable grant/contract number). The sponsors had no role in the design or conduct of this research.

\section{Compliance with ethical standards}

Conflict of interest The authors declare that they have no conflict of interest.

Ethical approval All procedures performed in studies involving human participants were in accordance with the ethical standards of the institutional and/or national research committee (Regional Ethics Committee in Gothenburg, Sweden) and with the 1964 Helsinki declaration and its later amendments or comparable ethical standards. For this type of study, formal consent was not required.

Open Access This article is distributed under the terms of the Creative Commons Attribution 4.0 International License (http:// creativecommons.org/licenses/by/4.0/), which permits unrestricted use, distribution, and reproduction in any medium, provided you give appropriate credit to the original author(s) and the source, provide a link to the Creative Commons license, and indicate if changes were made.

\section{References}

1. Adhiyaman V, Chattoopadyay I, Irshad F, Curran D, Abraham S (2017) Increasing incidence of chronic subdural haematoma in the elderly. QJM 110(6):375-378

2. Almenawer SA, Farrokhyar F, Hong C, Alhazzani W, Manoranjan B, Singh S et al (2014) Chronic subdural haematoma management: a systematic review and meta-analysis of 34829 patients. Ann Surg 259(3):449-457

3. Bartley A, Jakola AS, Bartek J Jr, Sundblom J, Förander P, Marklund N, Tisell M (2017) The Swedish study of irrigation-fluid temperature in the evacuation of chronic subdural hematoma (SIC!): study protocol for a multicenter randomized trial. Trials 18:471

4. Black S, Muller F (2010) On the effect of temperature on aqueous solubility of organic solids. Org Process Res Dev 14(3):661-665

5. Gazzeri R, Galarza M, Neroni M, Canova A, Refice GM, Esposito S (2007) Continuous subgaleal suction drainage for the treatment of chronic subdural haematoma. Acta Neurochir 149(5):487-493

6. Guilfoyle M, Hutchinson P, Santarius T (2017) Improved long-term survival with subdural drains following evacuation of chronic subdural haematoma. Acta Neurochir 159(5):903-905

7. Henning R, Kloster R (2007) Evacuation of chronic subdural haematomas followed by continuous inflow and outflow irrigation. Acta Neurochir 141(2):171-176

8. Ivamoto HS, Lemos HP, Atallah AN (2016) Surgical treatments for chronic subdural haematomas: a comprehensive systematic review. World Neurosurg 86:399-418

9. Liu W, Bakker NA, Groen RJ (2014) Chronic subdural hematoma: a systematic review and meta-analysis of surgical procedures. J Neurosurg 121(3):665-673

10. Miranda LB, Braxton E, Hobbs J, Quigley MR (2011) Chronic subdural hematoma in the elderly: not a benign disease. $\mathrm{J}$ Neurosurg 114(1):72-76

11. Schucht P, Fischer U, Fung C, Bernasconi C, Fichtner J, Beck J et al (2019) Follow-up computed tomography after evacuation of chronic subdural hematoma. J Engl J Med 380(12):1186-1187

12. Sjåvik K, Bartek J Jr, Sagberg LM, Henriksen ML, Gulati S, Jakola AS et al (2017) Assessment of drainage techniques for evacuation of chronic subdural hematoma: a consecutive population-based comparative cohort study. J Neurosurg. https://doi.org/10.3171/ 2016.12.JNS161713]

13. The Swedish National Board of Health online database of statistics (number of surgeries performed annually in Sweden) http://www. socialstyrelsen.se/statistik/statistikdatabas/operationerislutenvard. Accessed 10 Nov 2018

14. United Nations, Department of Economic and Social Affairs, Population Division (2015). World Population Ageing 2015 (ST/ ESA/SER.A/390). Accessed 8 Feb 2019

15. Watts DD, Trask A, Soeken K, Perdue P, Dols S, Kaufmann C (1998) Hypothermic coagulopathy in trauma: effect on varying levels of enzyme speed, platelet function and fibrinolytic activity. J Trauma 44(5):846-854

16. Yu GJ, Han CZ, Zhang M, Zuhang HT, Jiang (2009) Prolonged drainage reduces the recurrence of chronic subdural hematoma. Br J Neurosurg 23(6):606

Publisher's note Springer Nature remains neutral with regard to jurisdictional claims in published maps and institutional affiliations. 